\section{Methods for Casting Ancient Bone and Teeth for Viewing under the SEM}

Christopher W. Schmidt

Archeology and Forensics Laboratory

University of Indianapolis, Indianapolis, Indiana 46227

While using casts in place of original bones and teeth for study with the SEM is a rather common practice, periodic updates and introductions of unique casting methods help to keep the process of improving cast quality cumulative, limiting unnecessary repetition of ineffectual procedures. The following is a summary of casting methods that I have synthesized from several published reports as well as some that I have developed independently (e.g., Waters and Savage 1971; Scott 1982; Rose 1983; Gordon 1984; Teaford 1991; Buikstra and Ubelaker 1994; Schmidt, 1998). The study for which I first employed these methods consisted of hundreds of archeologically-derived human teeth dating from about 1,000 to around 10,000 years ago. The study required detailed views of dental surfaces magnified $500 X$ (Figure 1). The casts that I produced routinely had features that were distinct at magnifications of $2,000 \mathrm{X}$ and higher.

Before taking molds, the original teeth were washed with a $95 \%$ solution of ethyl alcohol (EtOH) and a soft-bristled toothbrush. As is a common practice in the curation of archeological material, some teeth were coated with polyvinylacetate (PVA). Teeth covered with this preservative proved to be very difficult to clean and had to be soaked in acetone until all of the PVA was removed. This process often lasted for several minutes. The teeth were checked periodically to see if they were tacky to the touch (while wearing a protective glove). Those teeth that were no longer tacky were considered clean, rinsed with $\mathrm{EtOH}$, and allowed to air dry.

A polyvinylsiloxane molding material, Coltene's President Light Body (available at most dental supply companies), was used for making the molds. The molding material consisted of a hardener and a base, both of which were forced from cartridges with a caulk-like gun into a mixing tip and then onto the specimen. The molding material was placed carefully on the tooth in order to minimize the trapping of air between it and the specimen. After $\vec{c}$ approximately 6 to 10 minutes, the mold was removed and placed $\frac{5}{\bar{v}}$ in an unsealed plastic bag where it was allowed to degas for a minimum of 24 hours. Degassing is the release of molecules re- $\frac{\rho}{\bar{z}}$ sulting from the chemical reaction between the hardener and $\stackrel{0}{0}$ base. Allowing the mold to degas for at least one day reduced the incidence of bubbling in the cast. Immediately prior to pouring the $\frac{3}{9}$ casts the degassed molds were washed with $\mathrm{EtOH}$ and allowed $\stackrel{0}{0}$ to dry.

The casts were made with an epoxy resin (4 to 1 Super Hard Epoxy Resin, available through Tap Plastics in California) that required a base-to-hardener ratio of $4: 1$ (determined gravitimetrically). The base and hardener were thoroughly manually mixed before the resin was placed into the molds. Disposable pipettes were used to place the resin into the mold in order to minimize the capture of air between the epoxy resin and the mold. The filled mold was placed into a manual centrifuge for approximately 15 to 30 seconds. This forced the resin deeply into the relief of the mold and helped to move bubbles away from the interface of the two materials. The casts were allowed to cure for 24 hours. Hardened casts were removed from the molds and were prepared

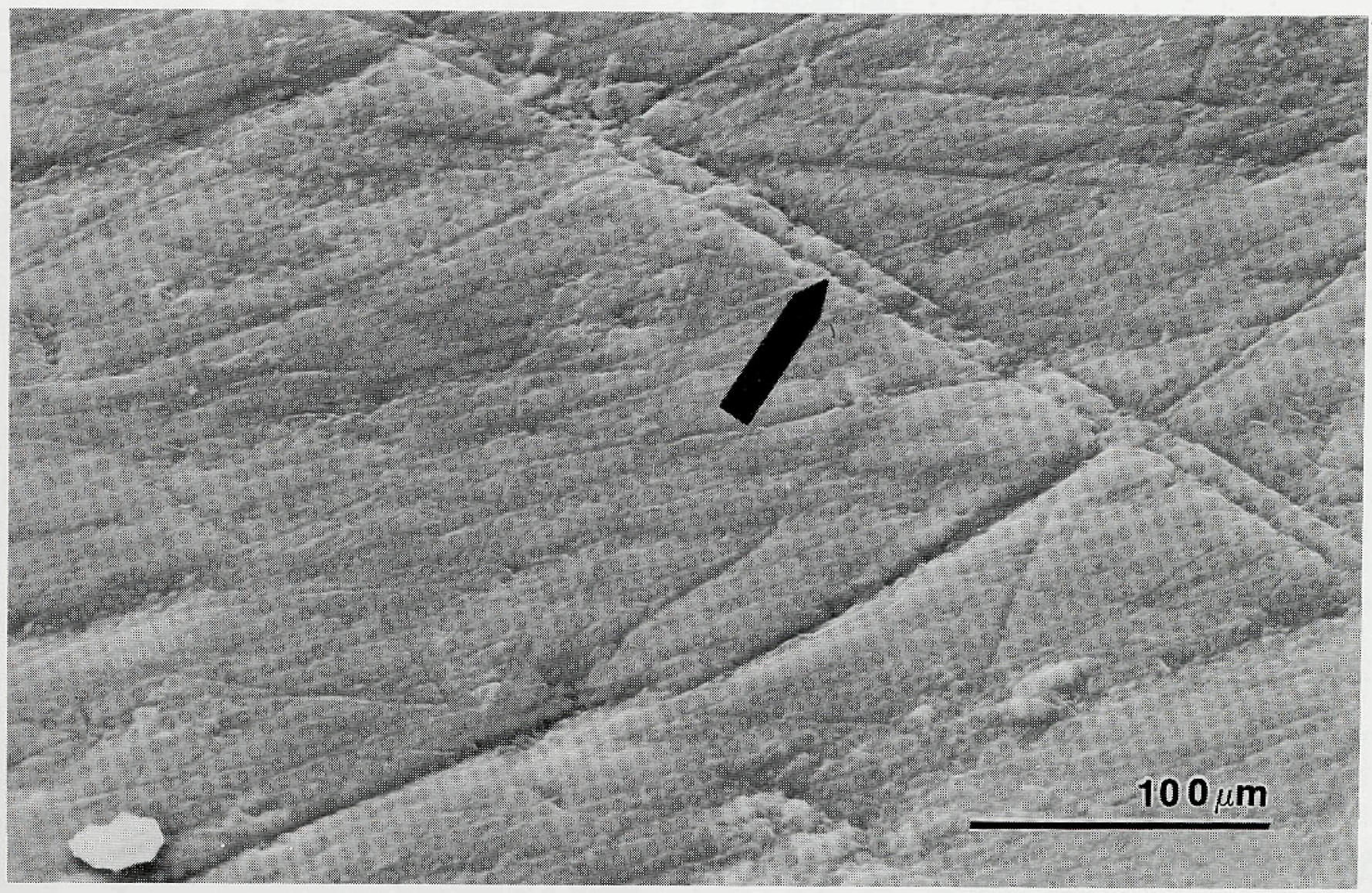

SEM of ancient tooth. Arrow points to scratch caused by grit in the diet. The individual to whom the tooth belonged probably consumed wild seeds, nuts, and perhaps, some maize. 
for scanning electron microscopy. They were rinsed in alcohol to remove debris that was sometimes picked up from the original by the mold. This happens very frequently with extremely brittle archeological and paleontological specimens. The casts are allowed to dry, sputter-coated and mounted on aluminum stubs with copper tape and conductive silver paint.

There are several advantages to using the particular materials employed here. President Jet is viscous enough to penetrate deep surface relief and it provides excellent detail of macroscopic and microscopic features. Super Hard Epoxy Resin replicates the mold with great detail and has very little tendency to bubble. Moreover, if the cast is no more than a few millimeters thick, the resin can be cut so that convenient sections of the cast can be viewed independently. This is not the case with all epoxy materials. Both materials are affordable (especially the resin) and can be easily obtained.

While using casts rather than originals has many advantages, such as protecting the original specimen and having a detailed copy of the original that is available for study at any time, there are some disadvantages. In general, casting procedures require many steps and thus allows plenty of opportunity for errors that damage the replicated cast or make it unusable. These errors include air bubbles in the molding or casting material, improper interactions between the bases and hardeners of the molding and or casting materials, dirt/dust accumulation on the mold or cast, etc.

Nonetheless, the methods described here help to minimize these hazards because they employ easy-to-use, high quality materials. Of the hundreds of casts that I have made following these methods, over $3 / 4$ are suitable for SEM study. By comparison, this percentage is two or three time higher than the success rate that I experienced with the combination of Reprosil molding material and Araldite epoxy, both of which are also high-quality materials. Overall, the casting methods described here are reliable and should be applicable to many areas of SEM-based osteological study.

\section{ACKNOWLEDGMENTS}

I would like to thank Debby Sherman of the EM Laboratory in Agriculture at Purdue University for her assistance in the development of these procedures. The study for which these methods were developed was funded by a grant from the Indiana Academy of Science (128-95).

\section{REFERENCES CITED}

Buikstra J.E., D.H. Ubelaker (1994) Standards for Data Collection

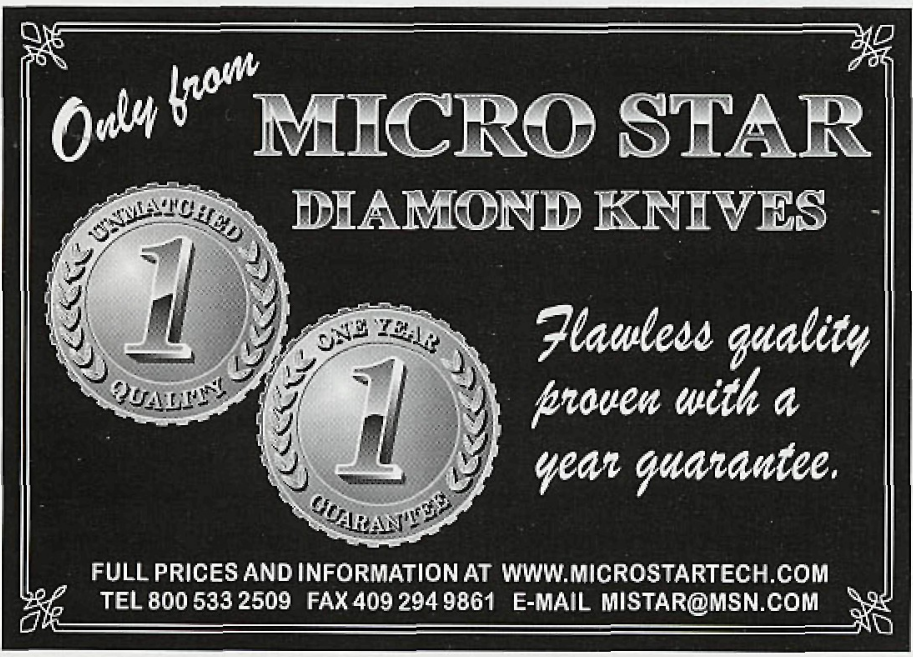

from Human Skeletal Remains. Arkansas Archaeological Survey Research Series No.44.

Gordon K.D. (1984) Pitting and bubbling artifacts in surface replicas made with silicone elastomers. J. Microsc. 134:183-188.

Rose J.J. (1983) A replication technique for scanning electron $\frac{}{6}$ microscopy: applications for anthropologists. Am. J. Phys. Anthropol. 62: 255-261

Schmidt C.W. (1998) Dietary Reconstruction in Prehistoric Humans from Indiana: An Analysis of Dental Macrowear, Dental Pathology, and Dental Microwear, Ph.D. Dissertation, Purdue University.

Scott E.C. (1982) Replica production for scanning electron microscopy: A test of materials suitable for use in field settings. J. Microsc. 125: 337-341

Teaford M.F. (1991) Dental Microwear: What can it tell us about diet and dental function? In Kelley M.A., Larsen C.S. Advances in Dental Anthropology. New York: Wiley-Liss. pp 341-356.

Waters B.T., D.E. Savage (1971) Making duplicates of small vertebrate fossils for teaching and for research collections. Curator $\frac{0}{\Phi}$ 14:123-132.
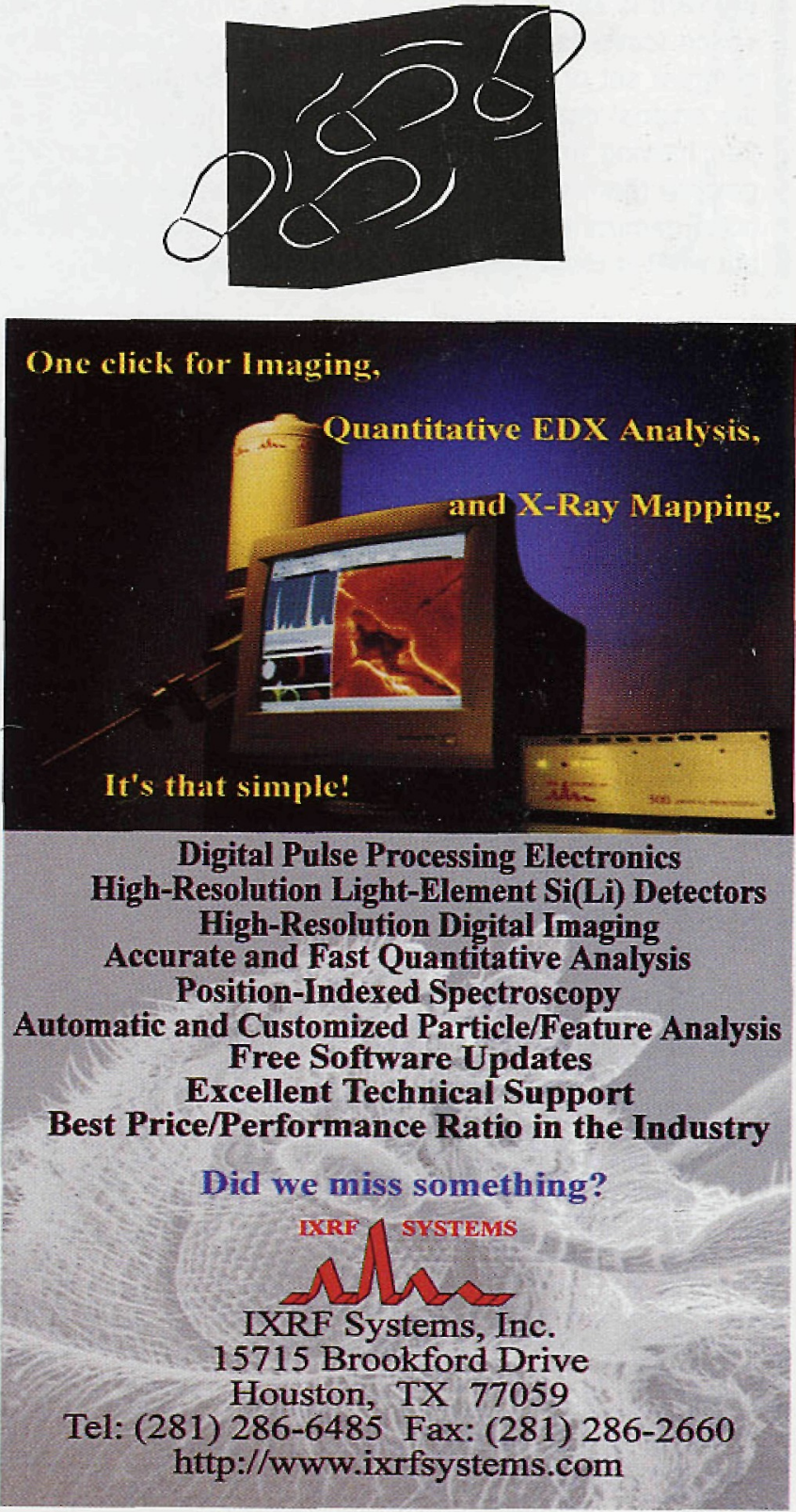